\title{
Assessment of high-school engineering education outreach program employing project-based learning in astronomy and bio-optics within a college setting
}

Sunil Dehipawala, Regina Sullivan, Raul Armendariz, Vazgen Shekoyan, George Tremberger, et al.

Sunil Dehipawala, Regina Sullivan, Raul Armendariz, Vazgen Shekoyan, George Tremberger Jr., David Lieberman, Tak Cheung, "Assessment of highschool engineering education outreach program employing project-based learning in astronomy and bio-optics within a college setting," Proc. SPIE 10741, Optics Education and Outreach V, 107410H (14 September 2018); doi: $10.1117 / 12.2320683$

Event: SPIE Optical Engineering + Applications, 2018, San Diego, California, United States 


\title{
Assessment of high-school engineering education outreach program employing project-based learning in astronomy and bio-optics within a college setting
}

\author{
Sunil Dehipawala, Regina Sullivan, Raul Armendariz, Vazgen Shekoyan, George \\ Tremberger, David Lieberman, \& Tak Cheung
}

CUNY Queensborough Community College, 222-05 56 ${ }^{\text {th }}$ Ave. Bayside, NY 11364

\begin{abstract}
$\underline{\text { ABSTRACT }}$
A college outreach engineering education program designed for high school students was implemented in a community college using the three principles of K-12 engineering education, namely, product design, science knowledge, and mindset development. The means of transforming a science question into a design driven one was illustrated using examples and projects from the fields of astronomy and bio-optics. The relevant scientific principles were presented by introducing various iterated designs in an interactive learning environment. A high school level research component using astronomy and bio-optics-related public data was also implemented to enrich the students' exposure to numerical processing techniques accessible in Microsoft Excel. Examples including solar events driven by magnetic field topology, cosmological images generated by IllustrisTNG Project, muon flux data, Killer T-cell motion, and wound assay cell migration are used in the teaching of iterated designs. A Path Diagram assessment model based on a LISREL computation scheme with learning intent and engineering mindset as latent variables was used to gauge the effectiveness of an implementation, the results of which would be used in the subsequent semester's implementation of the research component. The materials developed in a College Now course, where high school students take a research course at a community college, would be readily adaptable to other four-year college programs. The possible future incorporation of an introductory engineering course for high school student education, using the MIT low-cost $\$ 100$ muon detector and Fermi Lab QuarkNet muon scintillation detector, is discussed.
\end{abstract}

Keywords: design driven physics questions, iterated designs with constraints, magnetic field topology driven solar events, IllustrisTNG generated cosmological images, Killer T-cell motion, wound assay cell migration, cell population vitality, muon tomography.

\section{INTRODUCTION}

The Gen Z students, with birthdays starting from 1997 (Ref 1), are facing considerable challenges with declining literacy, numerical and problem solving skills according to the 2015 ETS report (Ref 2). Another 2015 survey shows that only $12 \%$ of the Gen $\mathrm{Z}$ students would learn by listening such as classroom environment (Ref $3)$. The automation impact on jobs have been reported as changes in six clusters (Ref 4, 5). The six reported clusters are doers, crafters, technicians, facilitators, providers, and solvers. A high school curriculum is the foundation for college education. It is beneficial for high school students to have some acquaintance in engineering concepts before entering a college engineering program. The three principles of K-12 engineering education, namely, design, science knowledge, and mindset development, explained by Linda Katehi (Chancellor of the University of California Davis and Chair of the Committee on K-12 Engineering Education in the National Academy of Engineering and National Research Council Center for Education) on her report to Congress (Ref 6), have been implemented in an outreach program employing project-based learning in 
astronomy and bio-optics within a college setting. Given that physics is a required subject for engineering education in most colleges, the implementation of using physics lessons to train high school students in the learning of design would need an independent assessment model.

The use of correlation in an education assessment model is a common practice where a task outcome could have a correlation with the corresponding task assignment for a class of students. When there are several correlations, the combined modeling approach could be less obvious. A Path Diagram assessment model based on a LISREL computation scheme has been used (Ref 7). The learning intent and engineering mindset as latent variables were used to gauge the effectiveness of the implementation, the results of which would be used in the subsequent semester's implementation of the research component.

\section{GENERAL DESIGN TRAINING}

The transformation of a regular physics problem to a design question can be done using the regular textbook physics problems, and has been discussed by us (Ref 8). The use of calculus facilitates the transformation such that the rate of change indicated by a trend on a graph could induce a design question with respect to a change. Examples include velocity versus reachable height, safety angle for leaning a ladder, static friction supporting pure rolling, kinetic friction versus ramp angle, etc. The first design could be an answer to a question using the trend in a simplified model while an iterated design would correspond to refined modeling with an addition constraint. For example, an instructor can take the basketball throwing example in Chapter 3 of Coletta (Physics Fundamentals Physics Curriculum \& Instruction; 2nd edition 2010) and ask how to deliver a first-aid package onto a higher balcony. A location versus angle (or speed) plot would yield a graphical trend with an understanding of the projectile motion knowledge (Ref 8). The use of complex notation is also interesting. The vertical direction kinematics equation would be an equation with an expression $\mathrm{y}=\mathrm{v} 0 * \mathrm{t}+$ $0.5 * \mathrm{a} * \mathrm{t} * \mathrm{t}+\mathrm{y} 0$. An equation of $\mathrm{y}=45 * \mathrm{t}+0.5 *(-9.8) * t * t-120$ represents an object thrown upward $45 \mathrm{~m} / \mathrm{s}$ from a well with a depth of $120 \mathrm{~m}$ from ground level. The equation has a solution of $\mathrm{t}=4.59+1.85^{*} \mathrm{sqrt}(-1)$ for reaching ground. A solution with sqrt(-1) component means that $45 *(4.59+1.85 * \operatorname{sqrt}(-1)+0.5 *(-9.8) *[4.59+$ $1.85^{*}$ sqrt(-1) $]^{*}\left[4.59+1.85^{*} \operatorname{sqrt}(-1)\right]=0$. It is trivial to show that the sqrt(-1) terms add up to zero and rewriting the numerical expression would give a modified depth for initial velocity value of $+45 \mathrm{~m} / \mathrm{s}$. The extra interpretation of a modified reachable height is a design innovation question to the students, made possible by the use of complex notation. Students having strong confidence in algebra should be able to handle the explanation with algebraic steps. The next question on the other solution of $\mathrm{t}=4.59-1.85 * \operatorname{sqrt}(-1)$ is interesting as well. The situation carries a similar interpretation of a modified depth reachable by a $-45 \mathrm{~m} / \mathrm{s}$ downward throwing situation.

Project-based learning is a holistic approach for the development of innovation in an engineering mindset. Project-based learning solves each individual problem when it arises. Examples in foundational mechanics, optics, and particle counting in a college outreach program for high school students have been presented (Ref 9). For example, the Stanford University Solar Research Center has a high school exercise on the understanding of magnetic reconnection using a rubber band (Ref 10). The exercise can be delivered with numeracy skill training. The tension of a rubber band can be calibrated with a spring scale such that the stretching energy could be modeled numerically. A triangular rubber band pattern could be the first design to illustrate the release of energy in magnetic reconnection. The first design has a physics explanation in terms of force equilibrium and stored potential energy changes. A subsequent modification to the first design, such as the use of a polygon pattern, would provide iteration opportunity to develop an engineering mindset. 


\section{PROJECT-BASED APPLIED RESEARCH}

Our Physics Department offers a college level astronomy course for high school students through Btech Program for those interested in Business. The hands-on labs include light refraction, telescope optics, laser interference/diffraction, element identification by spectroscopy, and an evening viewing using a Meade 16-in telescope. Our Department also offers a research course for high school students via our College Now Program. An applied research component for the understanding of solar events using cosmic ray and spacecraft public data has been implemented with deployable Microsoft Excel algorithms suitable for high school setting. An example of a solar reconnection event is described in this paper. The reports of the magnetics field topology influences on solar eruptions have been published (Ref 11,12$)$. The magnetic field topology can be studied in the magnetic charge topology (MCT) model in which magnetic pole forces can be described conceptually by the use of high school mathematics (Ref 13). The movie data of Reference Eleven is also available with individual time frames for further study (Ref 14). Image processing can be done using ImageJ, a free software from NIH USA (Ref 15). The first analysis design could be the analysis of the brightest signal spatial positions in terms of time frames so that a speed in numerical value of pixel per second can be obtained using Microsoft Excel. The next level of analysis design could be the change of brightness histogram, and brightness outcomes along the horizontal axis can be analyzed in terms of average and deviation. In a third level iterated design, the frequency would be modeled as Shannon information entropy, which equals $-\mathrm{p}^{*} \log (\mathrm{p})$ with $\mathrm{p}$ as the probability in communication engineering, for those high school students who are interested in image processing. A typical image is shown in Figure 1 after image conversion to 8 bits. A shift between two brightness histograms associated with the studied solar eruption is shown in Figure 2. The Shannon information entropy was about 5.3 bits for time frame at 11 $\mathrm{sec}$ and about 5.9 bits for time frame at $19 \mathrm{sec}$, with timing defined in Reference 14.

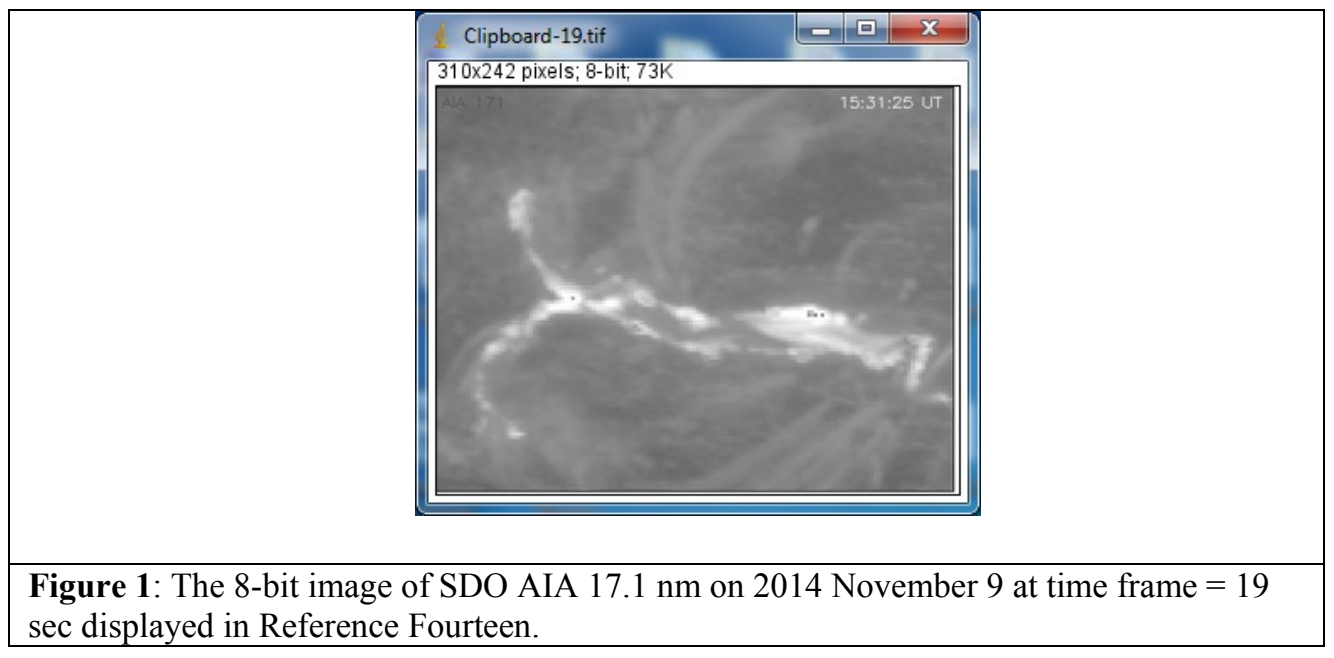




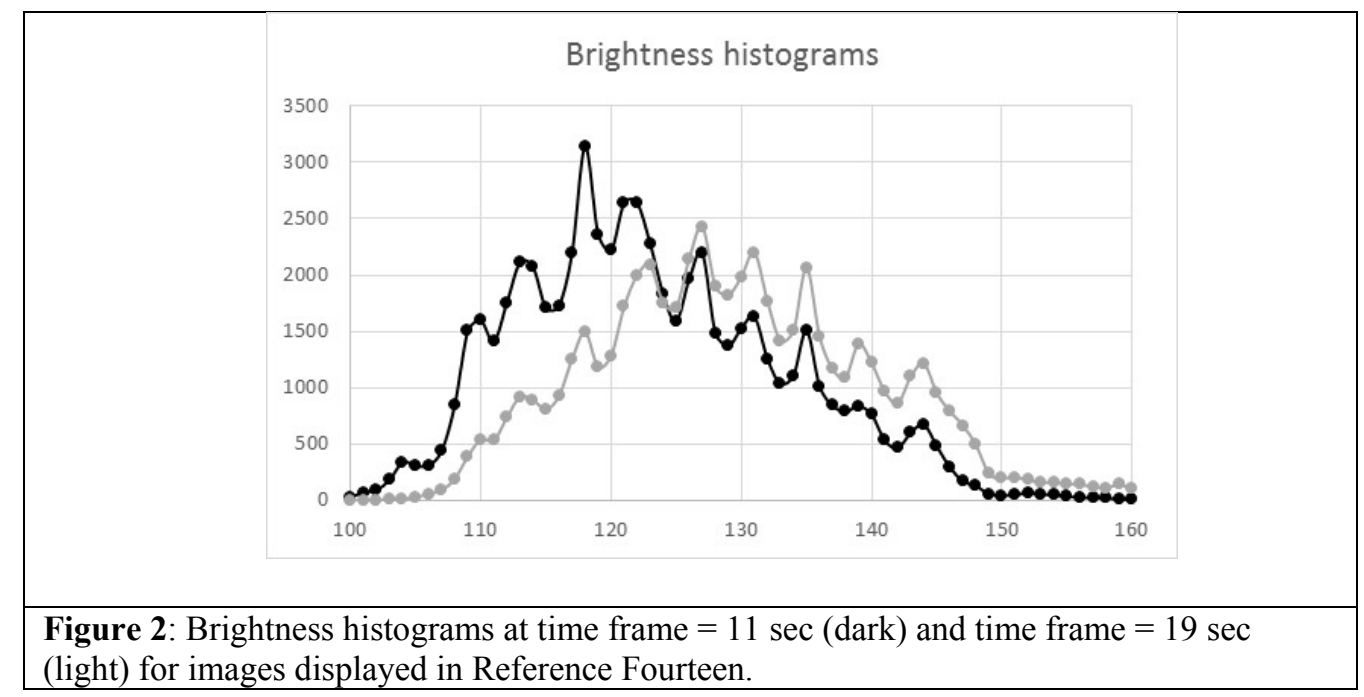

An applied research component for the understanding of Killer T-cell motion is described in this paper. The Killer T-cell research was published and the movie data "Killer T Cell: The Cancer Assassin" is available from Cambridge University or Youtube for download (Ref 16, 17). Correlated random cell motion analysis had been reported by us in 2009 based on the analysis of tetraploid cancer cell motion video data (Ref 18), and also by other researchers in 2017 and 2018 based on stem cell motion video data (Ref 19, 20). A high school level analysis method has been developed. A single frame in the Killer T-cell video, Figure 3, can be analyzed with simple kinematics. The first design could be to collect data on the right edge positions of the Killer T-cell and the left edge positions of the cancer cell versus time, shown in Figure 4. The upper dataset representing the Killer T-cell had a coefficient of variation CV of about 0.084 while the lower dataset representing the cancer cell had a CV of about of 0.083 . A second iterated design would be the separation of the motion analysis into two parts. At time $=55 \mathrm{sec}$, the Killer T-cell exhibited a temporary protrusion at the unengaged free edge, shown in Figure 5. Whether this extra protrusion could be viewed as an extra anchor of the Killer T-cell in the tug of war with the cancer cell is an interesting question when more videos are available. We know that cancer cells can use integrin related mechanism to migrate to the brain through the cerebrospinal fluid system without crossing the blood brain barrier (Ref 21). For the time duration preceding the free edge protrusion, the Killer T-cell dataset $\mathrm{CV}$ was about 0.065 and the cancer cell dataset $\mathrm{CV}$ was about 0.058 . For the time period after the free edge protrusion, the Killer T-cell dataset CV was about 0.030 and the cancer cell dataset CV was about 0.041 . The cancer cell motion showed more variation when compared to the Killer T-cell motion as if the Killer T-cell protrusion was effective in enhancing the Killer T-cell gripping of the cancer cell. A third iterated design would be the Shannon entropy computation in terms of brightness histogram variation, which is expected to be related to the internal cellular processes such as actin movement (Ref 22). Although Fluorescence microscopy data collection is not on our outreach program course in Spring Semester 2018, about 20\% high school students expressed interests in bio-optics learning and returned in Summer Semester 2018 for a second opportunity. 


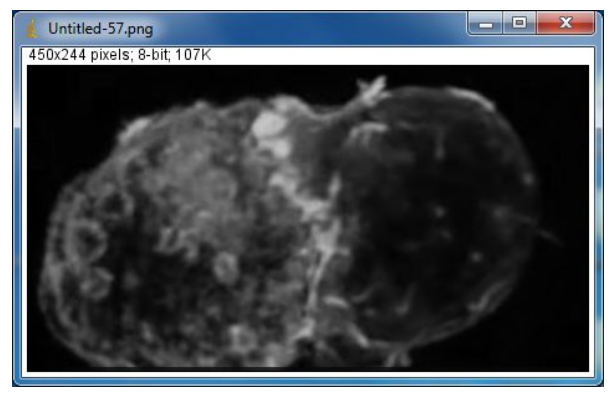

Figure 3: An 8-bit image representing the interaction between a Killer T-cell and cancer cell. The Killer T-cell is on the right and the cancer cell is on the left at time frame $=57 \mathrm{sec}$.

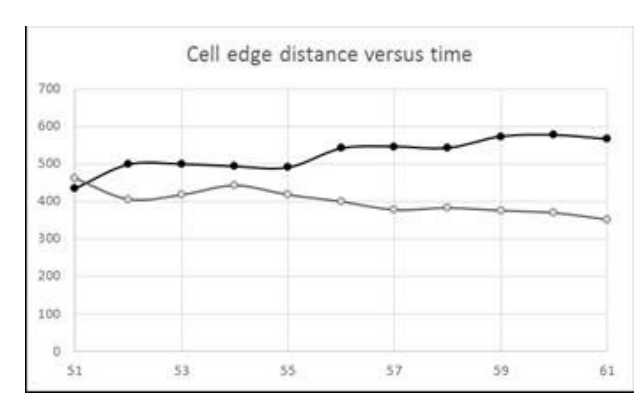

Figure 4: Cell edge distance (arb unit), versus time (sec), upper T-cell, lower cancer cell

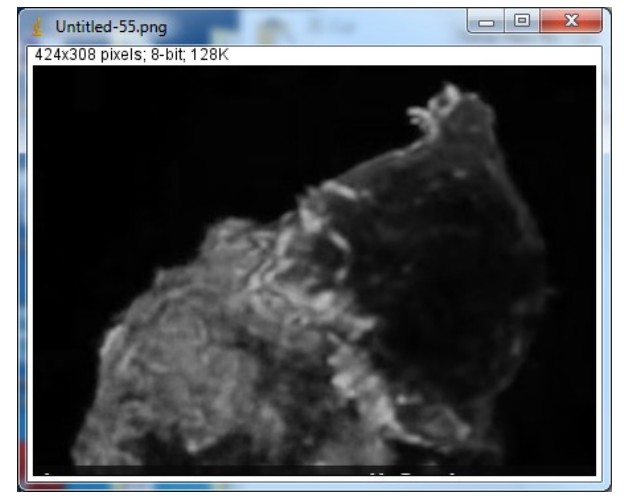

Figure 5: An 8-bit image representing the interaction between a Killer T-cell and cancer cell. The Killer T-cell is on the right and the cancer cell is on the left at time frame $=55 \mathrm{sec}$, timing defined in Reference Seventeen.

Another analysis of cell migration in a wound assay would offer a deeper inquiry of cell collective motion beyond a first design with velocity related questions. A wound assay movie from Newcastle University has been available in the public domain (Ref 23). A typical frame, with brightness contrast processed by ImageJ for easy viewing, is shown in Figure 6. A typical raw brightness profile, a single-pixel width digitization window without brightness contrast processing, along a studied image is shown in Figure 7 in which the edge 
positions can be obtained for velocity related questions. A second iterated design could involve a study of the brightness variation in terms of the coefficient of variation values and whether the brightness variation is symmetrical in the right side and left side of the gap in the image. A third iterated design could involve a study of the brightness percent increment when comparing two adjacent pixels, defined as (A2-A1)/A2 in Excel notation, shown in Figure 8. The histogram of the brightness percent increment is shown in Figure 9 in which the width would be the volatility. The volatility of the gap sub-region brightness would be much lower when compared to the volatility of the left or right sub-region adjacent to the gap. The cell population vitality could be represented by the time dependent volatility values, given that cell vitality and viability are both important measures in regenerative medicine (Ref 24). Measuring vitality without using fluorescent signals could be an improvement (Ref 25). One of the Summer Semester projects was "Carbon nanotube effect on breast cancer cell wound assay healing study" using established methods (Ref 26). Our fiber optics equipment, including phase contrast microscopy and sub-micron probes, have also been used for near field bio-optics experiments with high school students (Ref 27).

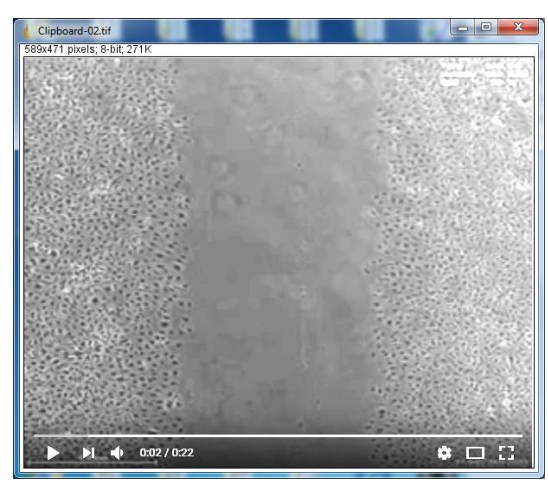

Figure 6: An image extracted from the studied wound assay video of Reference 23.

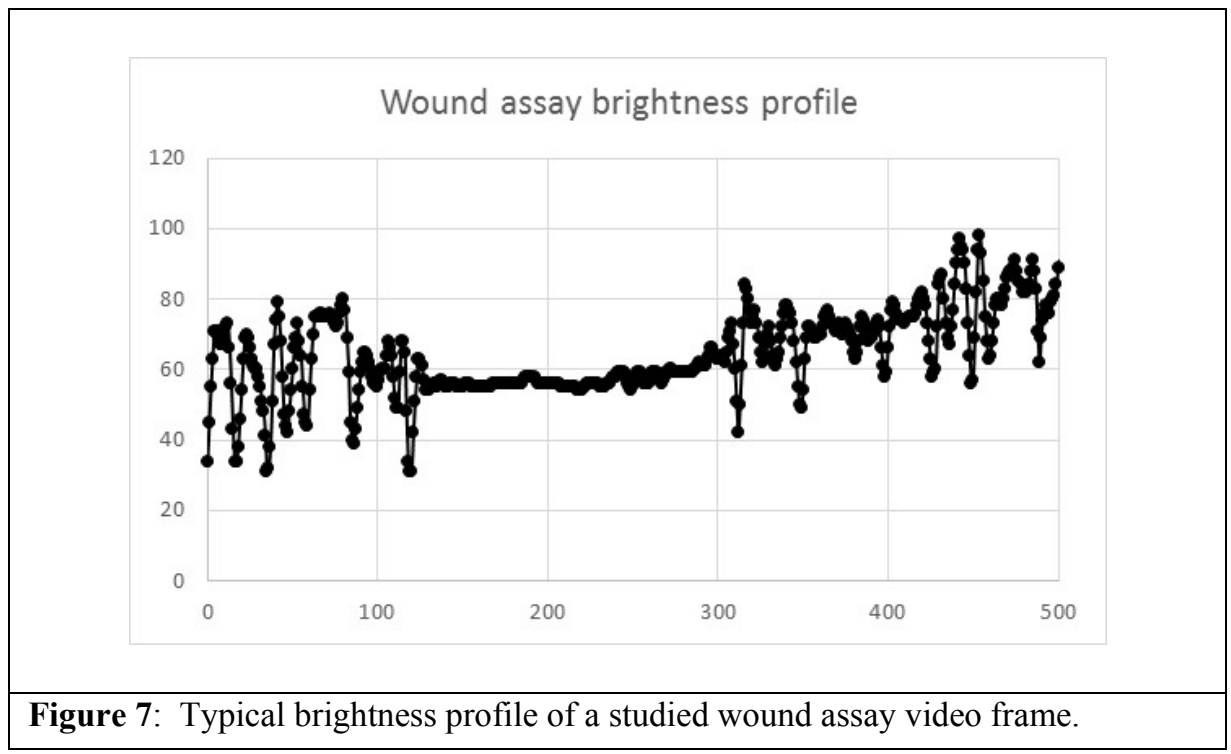



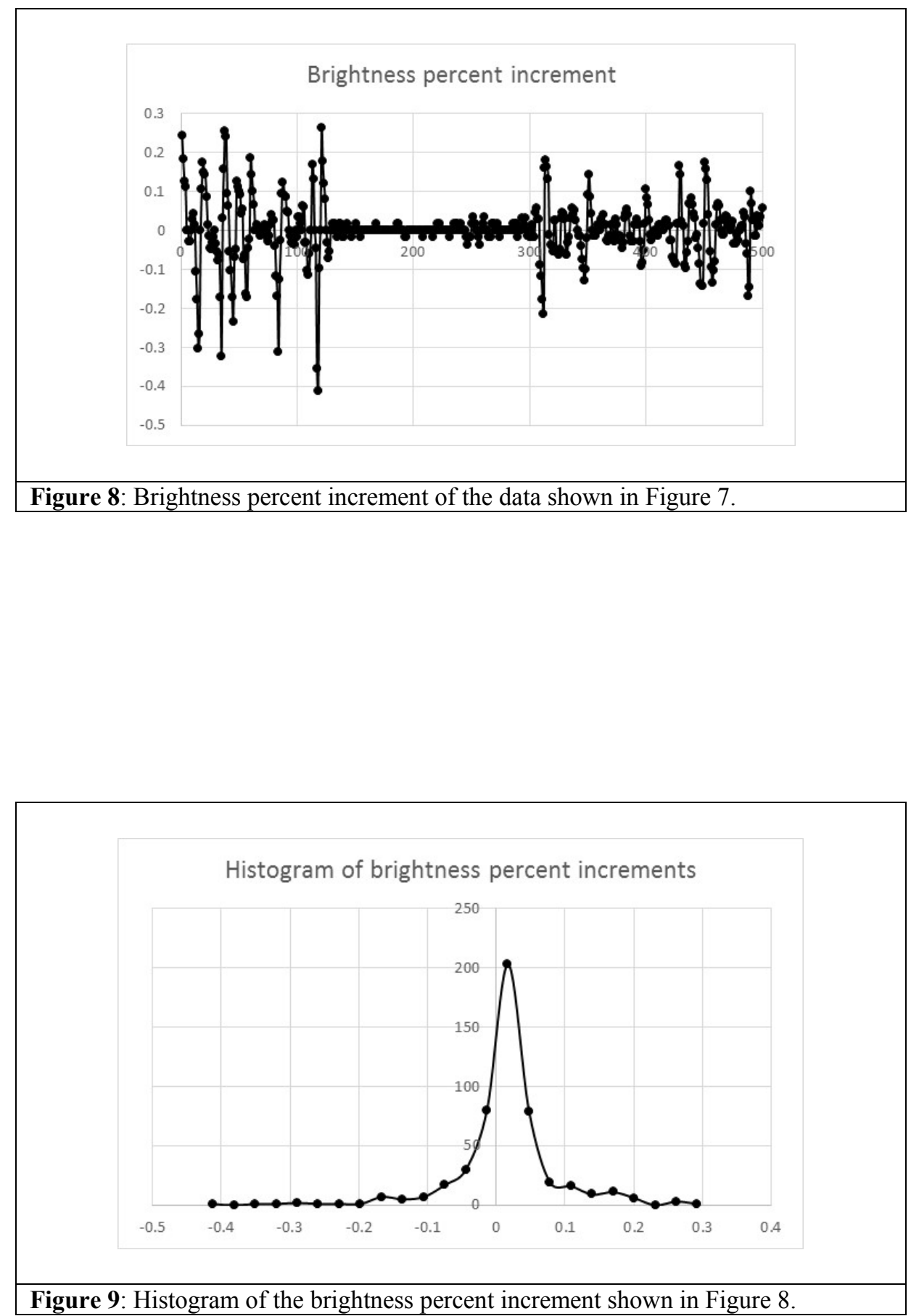

Proc. of SPIE Vol. 10741 107410H-7

Downloaded From: https://www.spiedigitallibrary.org/conference-proceedings-of-spie on 26 Apr 2023 Terms of Use: https://www.spiedigitallibrary.org/terms-of-use 


\section{EDUCATION ASSESSMENT}

Regression analysis, which requires an independent variable, has been an important data analysis procedure in the STEM curriculum. The practice of analyzing several variables in terms of multiple regression and latent variable techniques would give a holistic perspective. An example that addresses the application of latent variable modeling to assess an outreach program for high school students using the LISREL software is presented. A start step by step instruction set for running LISREL on Windows had been published by us (Ref 28), and the steps are included here for easy reference. The step by step procedure for using the free LISREL student version are described as the followings. (1) Calculate the data and put in the random noise as columns in a spreadsheet, with first row containing variable names, then save as CSV format, then put the CSV in a folder. (2) Open LISREL, File/import data, open CSV file and save as LISREL system file, computer would echo the variable names. (3) File/new/path/, give a name for the path diagram of the postulated latent variable relationship to the data. (4) Setup/variable, click add-variable, click system file, click browse for system file, then computer would echo the variable names, then click add-latent, "int1" and "int2" (in this case). (5) Draw path, by dragging the items displayed on the left panel. (6) Select basic model, standard solution, just above the path diagram. (7) Setup/ build lisrel syntax and then File/run.

An example of education assessment is displayed in Figure 10. The "intent" latent variable loads onto the calc1 and calc2 data from an assessment task in solving a physics problem using calculations. The "mindset" latent variable loads onto the design- 1 and design-2 data from an assessment task in a research project. The path diagram example could serve as an assessment of "intent of learning" with an engineering mindset. The data used is displayed in Figure 11 for readers interested in further analysis in terms of alternative path diagrams, covariate sensitivity, etc.

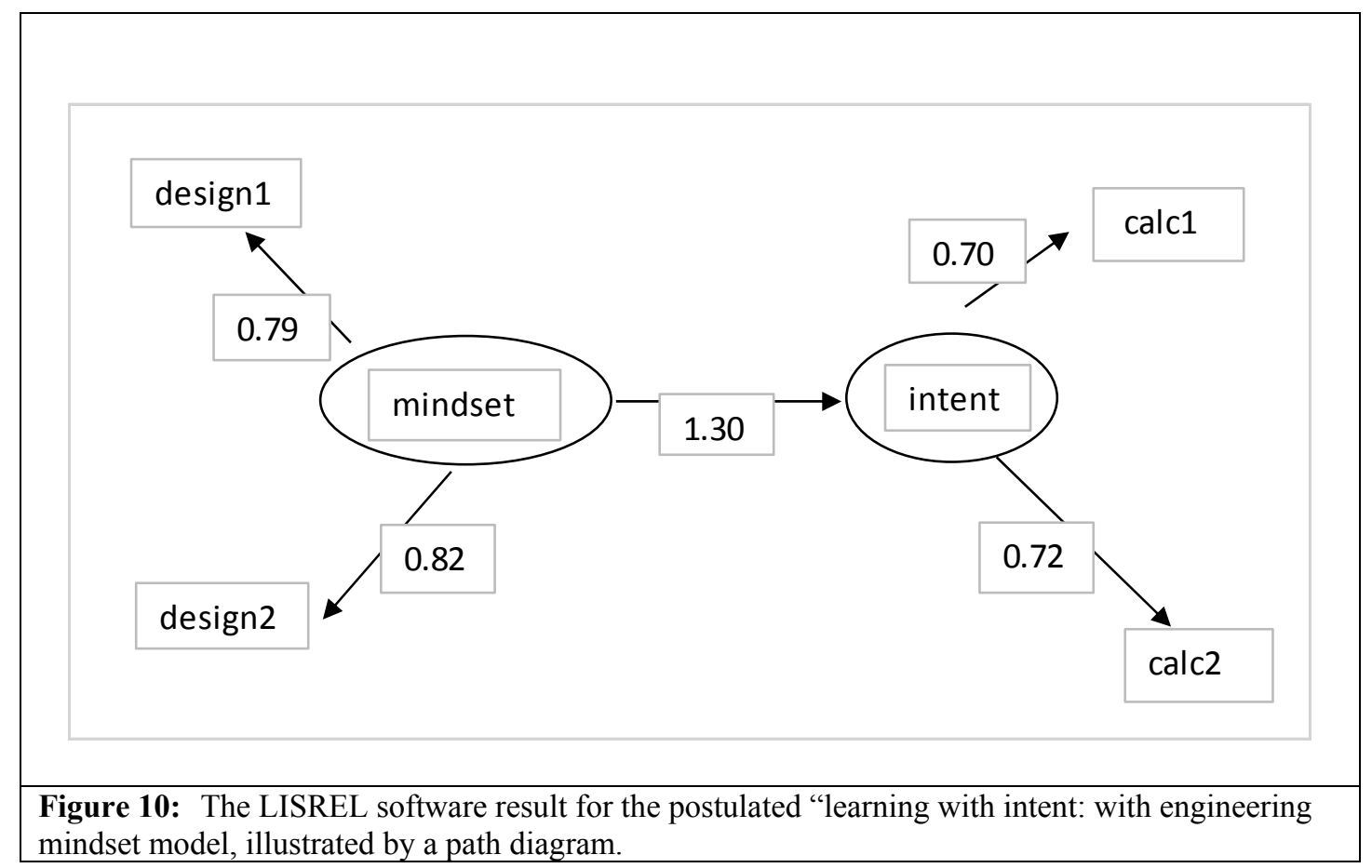




\begin{tabular}{|c|c|c|c|c|c|c|c|}
\hline design-1 & design-2 & calc-1 & calc-2 & design-1 & design-2 & calc-1 & calc-2 \\
\hline 97 & 96 & 65 & 82 & 75 & 65 & 15 & 51 \\
\hline 88 & 80 & 30 & 76 & 65 & 59 & 10 & 51 \\
\hline 75 & 77 & 30 & 76 & 78 & 73 & 42 & 45 \\
\hline 85 & 79 & 25 & 76 & 62 & 59 & 19 & 42 \\
\hline 79 & 84 & 50 & 75 & 62 & 58 & 15 & 40 \\
\hline 85 & 79 & 25 & 72 & 60 & 53 & 15 & 38 \\
\hline 83 & 77 & 32 & 70 & 60 & 53 & 6 & 38 \\
\hline 86 & 87 & 57 & 69 & 60 & 51 & 12 & 38 \\
\hline 85 & 73 & 25 & 66 & 30 & 14 & 15 & 26 \\
\hline 60 & 30 & 25 & 63 & 17 & 9 & 10 & 30 \\
\hline 82 & 66 & 15 & 53 & 10 & 5 & 8 & 25 \\
\hline
\end{tabular}

\section{DISCUSSION}

The incorporation of an introductory engineering course for high school student education using lowcost optics, such as the $\$ 100$ muon monitoring device from MIT, is not yet practical in our opinion (Ref 29, 30). The high school students in our College Now program showed difficulties in handling the tiny electrical components. And even when experienced faculty helped out in the circuit construction, some electrical components were still not available as of July 2018 with a purchase order dated March 2018. Our Queensborough Community College QCC is a member of the Fermi Lab QuarkNet. We have received QuarkNet equipment to enable us to build muon scintillation detectors to characterize cosmic ray events for high school deployment so that students can be enriched directly in their high school environment (Ref 31). Even without the MIT \$100 muon detector, high school students can still do data analysis on the muon flux collected by our muon detectors using the QuarkNet technology. The fluctuation of muon flux is a more subtle concept when compared to solar brightness variation driven by magnetic field topology or image brightness variation driven by actin related cell morphology. Nevertheless a muon flux phenomenon can still be parametrized with numerical values. An example of muon flux collected by a Fermi Lab QuarkNet muon scintillation detector at QCC Physics is shown in Figure 12, with a first muon flux suppression occurred at New York local time 23:00:54 September 10 2017. The coefficient of variation CV for a duration of 100 data points was computed, and the results for several durations are shown in Figure 13. The gradual increase of the CV values preceding the first muon flux suppression event, registered by a QuarkNet muon scintillation detector at QCC, would suggest a volatility mechanism with a prolonged duration, within the high school level concepts of average and standard deviation (Ref 32). The cosmic ray data analysis using muon and neutron data from other stations is important to keep up the various interests of high school students when they are asked to polish many scintillation slabs for photon noise reduction at each slab's boundary, and our data are uploaded to Fermi Lab for their staff to analyze. 


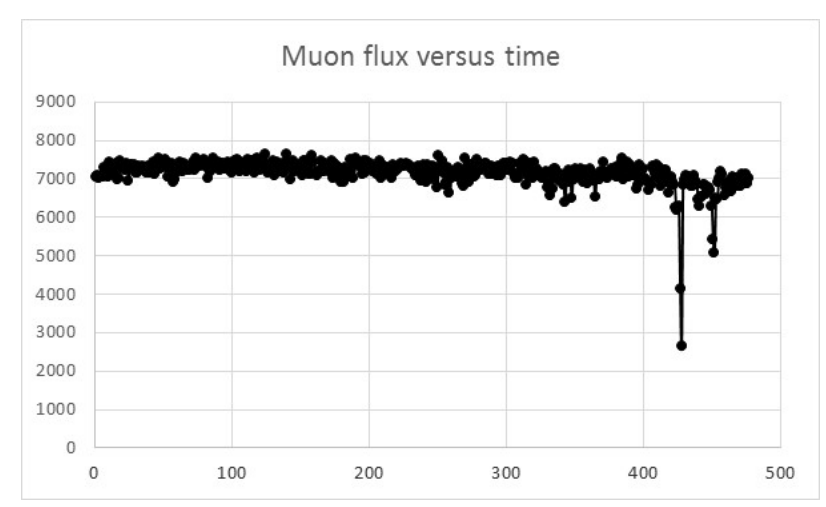

Figure 12: Muon flux count vs. time (every 5 min) starting from 9/9/2017 at 11:25:54 local time.

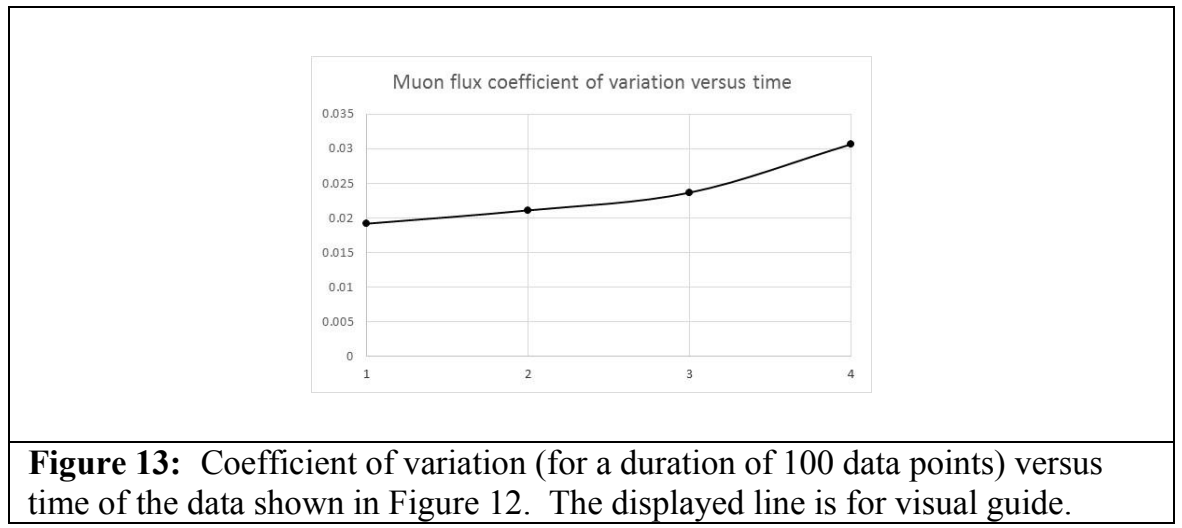

There are usually five to ten high school students each year who are interested in doing advanced projects to support their applications to the most competitive colleges. The Yale University crumpled paper fractal example for high school students was found to integrate well for students using the ImageJ fractal analysis method (Ref 33). For example, the evaluation of the dark matter density simulation results can be performed in ImageJ (Ref 34). The simulation images of "Reference 34 - Figure 2" were converted to 8-bit resolution, shown in Figure 14. The fractal dimension and entropy values of the simulation images were computed, shown in Figure 15. It is interesting to note that the fractal dimension of the dark matter density upon extrapolation to earliest detectable epoch would be around 1.97, consistent with a correlated randomness interpretation of dark matter density in the published cosmological galaxy formation simulation study in the IllustrisTNG Project. 


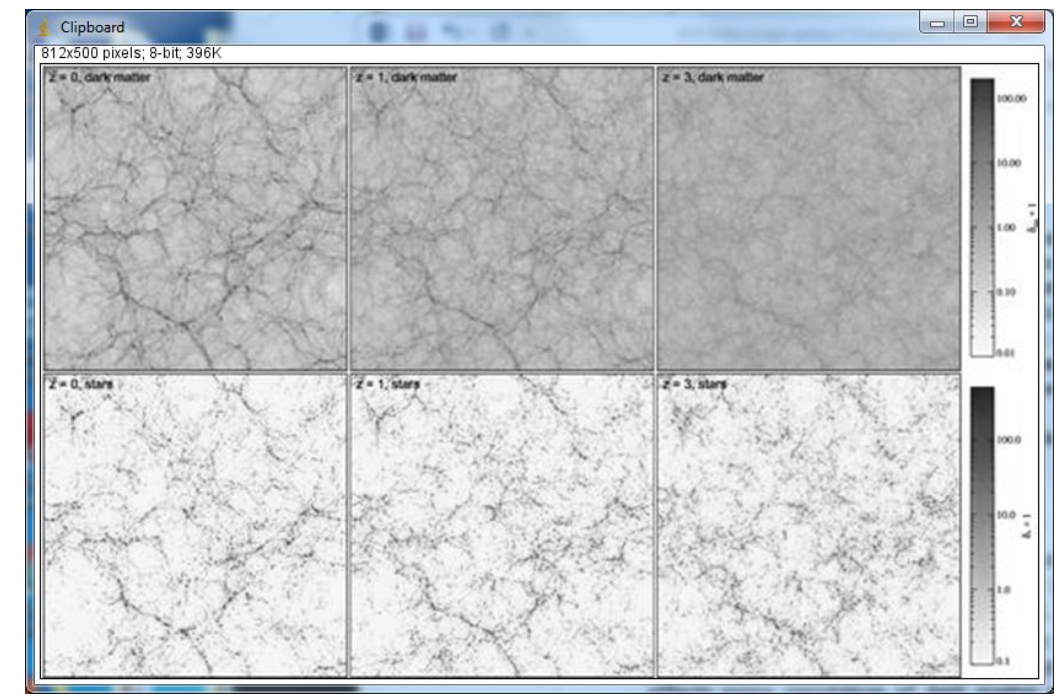

Figure 14: The 8-bit conversion of Ref-34 simulation images for dark matter density (upper) and stellar matter density (lower) at three different redshifts of 0,1 , and 3.

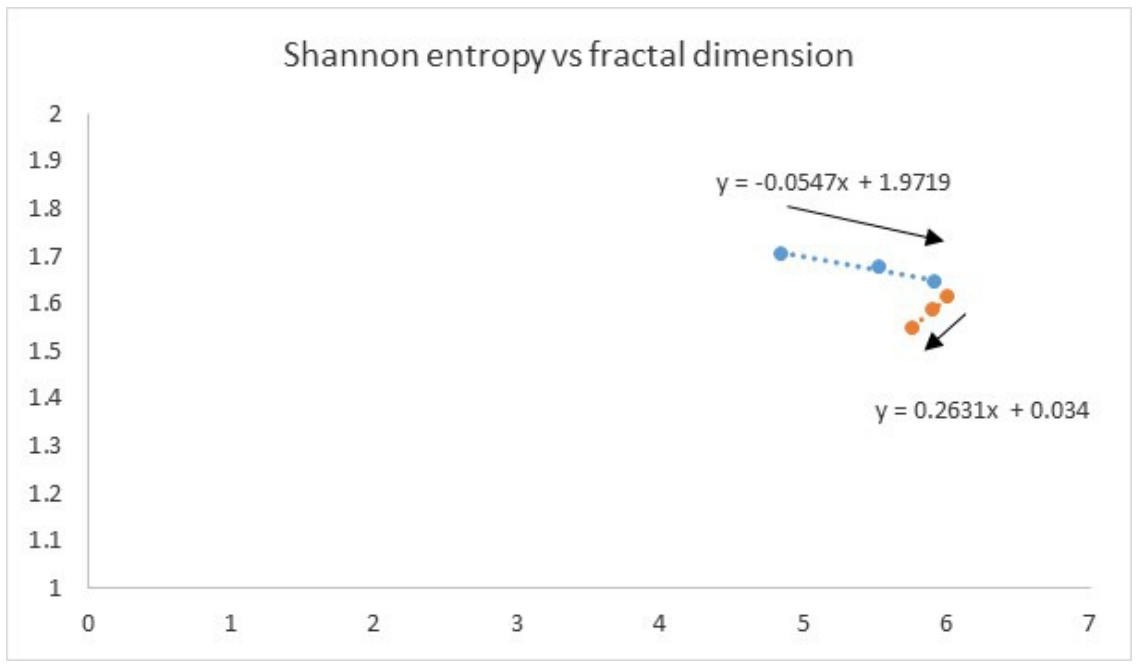

Figure 15: Fractal dimension value (y-axis) versus Shannon entropy value (x-axis), with upper data group representing dark matter density (adj R-sq $=0.93$ ) and the lower data group representing stellar matter density (adj R-sq $=0.99$ ) for three different redshifts of 0,1 , and 3 . The arrow directions indicate time direction from high redshift to low redshift.

Fractal dimension analysis can also be applied to bio-optics image analysis when high school students show interests. For example, a Liver Wildtype image and a Liver TEAD2-DN image with hematoxylin and eosin staining were available (Ref 35,36 ). The 8-bit conversion images are shown in Figure 16. The fractal dimension values were computed using ImageJ. The Wildtype image brightness showed a fractal dimension of 
1.726 with $\mathrm{CV}=0.231$. The DN image brightness showed a fractal dimension of 1.718 with $\mathrm{CV}=0.227$. The Wildtype brightness had a higher fractal dimension and $\mathrm{CV}$, suggesting that an expected higher metabolic activity in the Wildtype could be measured with brightness fluctuation parameters like fractal dimension and CV.

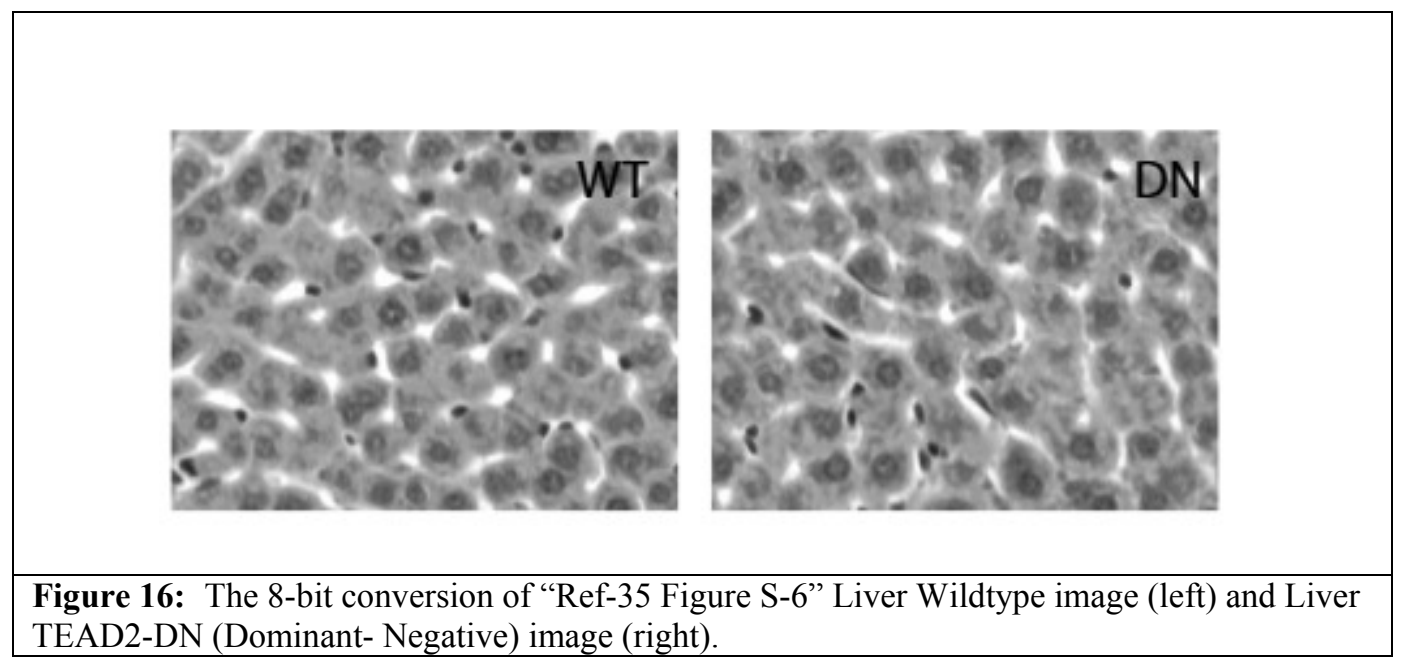

Project based learning in astronomy and bio-optics in an outreach program for high school students would improve numeracy skill and the iterated designs would develop engineering mindset. A latent variable assessment model would offer a checking guidance concerning the outreach program effectiveness, and also would be used in the subsequent semester's implementation. The fact that only $12 \%$ of the Gen $\mathrm{Z}$ students would learn by listening, such as classroom environment, demands new teaching methods with more hands-on exercises (Ref 3). The materials developed in a College Now course, where high school students take a research course at a community college, would be readily adaptable to other four-year college programs. A recent study shows that the Gen $\mathrm{Z}$ students who are high frequency digital media users are at risk in developing symptoms of Attention-Deficit/Hyperactivity Disorder ADHD (Ref 37). Another recent report also found that the use of digital technology would counteract the so called "Attention Enhancement Effects of Green Spaces" due to the fatigue in the capacity of paying attention in the context of Attention Restoration Theory (Ref 38). Whether the exercises described above could alleviate the ADHD symptoms is an interesting question for future studies.

For those high school students having much higher SAT scores than most STEM students in a community college, the implementation of research projects for them in a subsequent semester is challenging since they all aim to continue onto competitive colleges. Our photomultiplier tube (PMT) electronics and nanosecond pulse instrumentation in the muon detector construction project are handled by college students with sufficient background, while the cutting and polishing tasks of plastic slabs doped with fluorescent hydrocarbons for scintillation are available for the high school students. Our confocal laser microscopy, scanning tunneling microscopy, infrared spectroscopy, etc. used in the carbon nanotube effect on breast cancer cell project are handled by experienced college students while our stereo microscope and wound assay healing instrumentation are available to the high school students. The continued analysis of data in the public domain in the Spring Semester to the Summer Semester would uplift the self-confidence of a high school student when he/she receives an instrumentation which requires minimal skill, and also would remove the stress when a data collection fails to generate usable data for analysis during the short summer session. However there was one exceptional case that QCC data could supplement online data with contributions from high school students having high SAT scores. The QCC muon detectors offer a unique opportunity for high school students to appreciate high energy physics instrumentation such as the plastic scintillation detector calibration using the CERN muon beamline (Ref 39). A QCC muon detector response function would convolute with a scintillator light pulse to yield a waveform displayed on a gigahertz scope. A muon going through two consecutive detectors would generate two light 
pulses such that a third pulse from a third consecutive detector could become a predictable event, with proper oscilloscope triggering. The predictability would be violated when there is an additional layer that dissipates the muon energy. A muon could leave the second detector and lose extra energy in the additional layer before entering the third detector, shown in Figure 17. In other words the violation of the predictability of the third detector could be used to infer the presence of an additional layer, the principle of muon tomography. An understanding of the de-convolution mathematics was a bit difficult for the participating high school students so the pulse characteristics (such as height, width, area, etc) were used to select an event that showed two similar pulses on the first two detectors. The third detector response prediction with and without an additional layer were tested. The QCC triplet detectors, each equipped with a 0.75 inch plastic scintillation slab, was able to detect the presence of an additional plastic scintillator slab with a thickness of 2.5 inches at about $40 \%$ signal attenuation (measured/predicted $=0.7$ ), shown in Figure 18. Setting an attenuation detection limit at $10 \%$ would give $\mathrm{S} / \mathrm{N}$ around 3, which could be further improved with statistics. The extra scintillation slab can be viewed as a latent detector with no readable output, and the last detector voltage output could be used to infer the existence of a latent detector. Besides showing the muon tomography principle and possible applications, the investigation of slow muon with a stacking of multiple detectors at various separations given a thick attenuation layer would be appropriate at the high school level (Ref 40). Adding a magnet could demonstrate the principle of the Muon g-2 experiment as well for the high school students (Ref 41). Detailed study of each detector's signal could be used to locate the muon directions of some particular events relative to a star such as Polaris, and also be archived as a supplement database to muon archival data in terms of counts per second at various water meter equivalent depths for cosmic ray and solar physics studies in astronomy. Basically the grunt work of polishing and testing the recycled plastic scintillation slabs and PMT equipment from Fermi Lab are unavoidable, and the outreach instructors need to balance the grunt work and the development of the mindset of a young investigator.

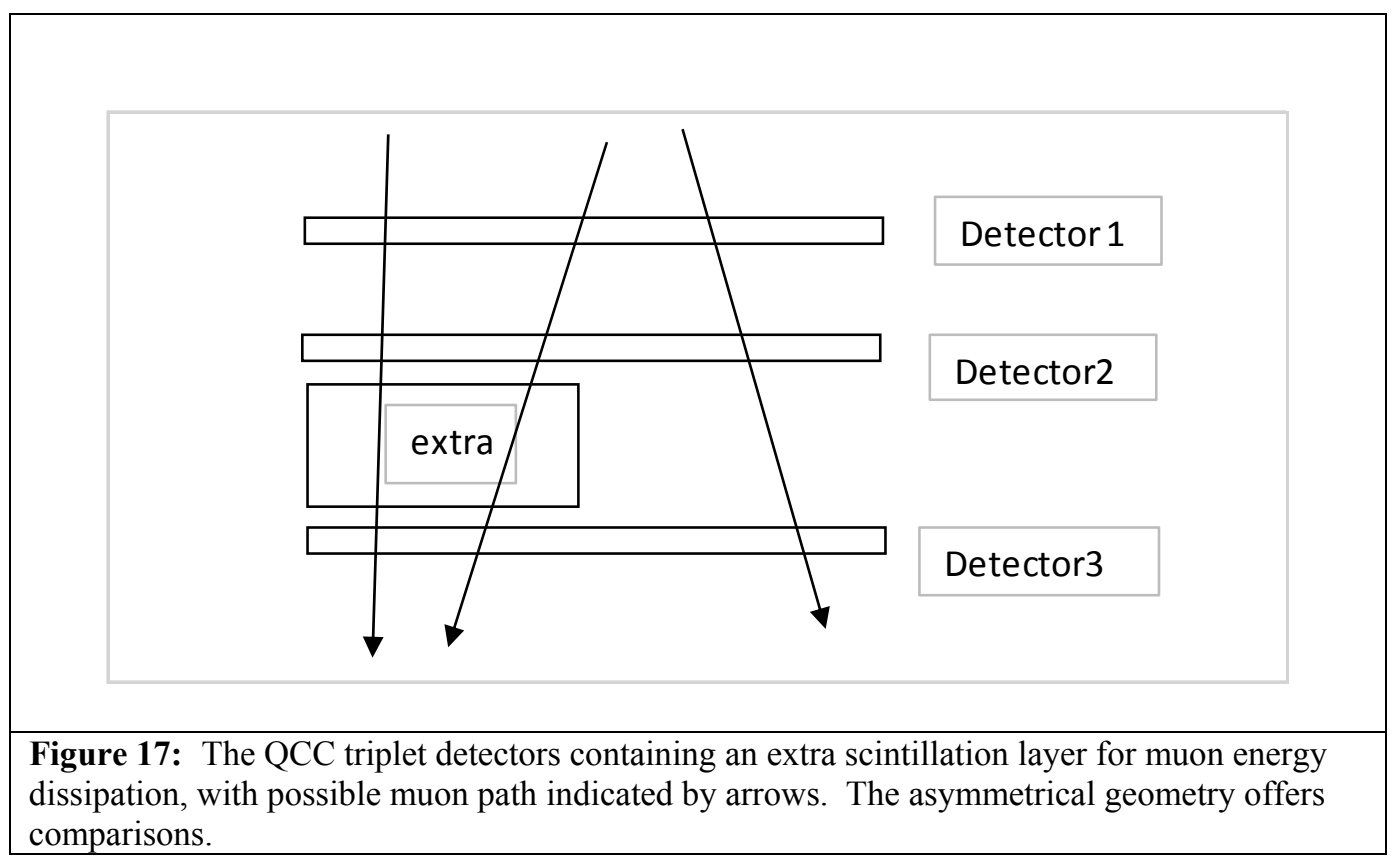

Proc. of SPIE Vol. 10741 107410H-13 


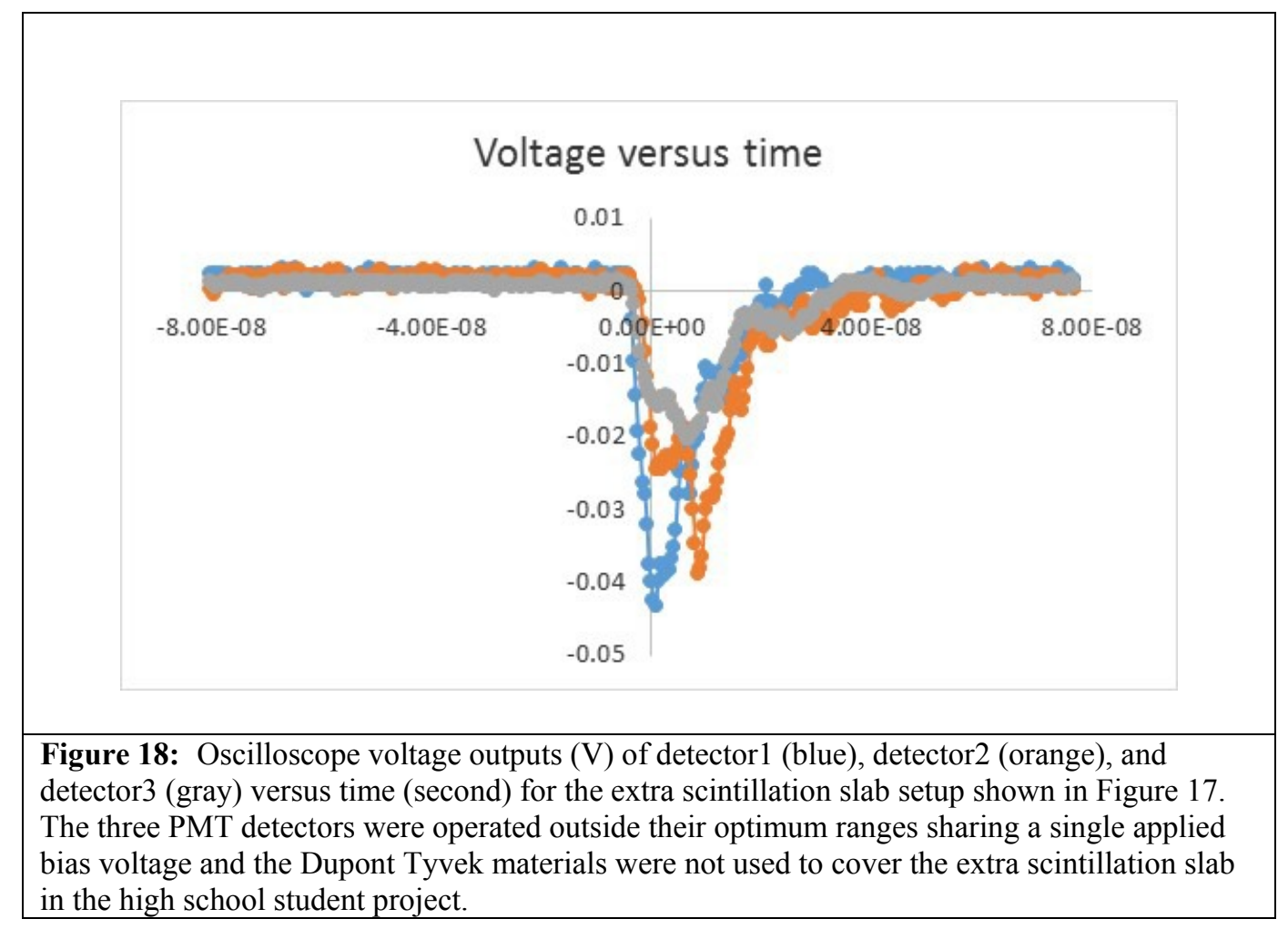

The design training is crucial for the development of an engineering mindset, starting from the first semester research course to the subsequent summer session research project for the participating high school students, and learning assessment will enable continued improvement in an outreach program. Grunt tasks such as polishing recycled scintillation slabs, testing previously used photomultiplier tubes, preparing Petri dish cancer cells for wound assay healing migration imaging, capturing images from standard optical set ups, aligning samples for measurements in standard bio-optics equipment, running ftp data download and processing data, programming code migration from FORTRAN to a newer language such as MS Excel-VBA in the same operating system, looping through parameters inside stored simulation-procedure, etc., are the skills required at the technician level without much scientific critical thinking. An outreach programs that includes the training of engineers and scientists must contain iterated designs, new insights or alternative evaluative criteria, and hypothesis generation. In other words, the grunt tasks that are replaceable by the advancing AI technology in five years or so must be supplemented by scientific critical thinking tasks in an outreach program that recruits motivated high school students to select STEM careers beyond the technician level (Ref 42). Even a high school student project on an ecology study of bacteria in the near-by park that used a standard Biolog instrumentation could be supplemented with a literature survey of Matrix-Assisted Laser Desorption Ionization- Time of Flight Mass Spectrometry, and be expected to provide some judgment linked to critical thinking in bio-engineering advances (Ref 43). The learning assessment rubric in our outreach program second semester research project has four deliverables, (1) delivery of an acceptable product at a technician level, (2) surveying of literature with judgment linked to critical thinking as a young investigator/solver (Ref 4, 5), (3) discovery of new findings or alternative evaluative criteria to supplement published results, (4) generating design hypothesis with a cost estimation of consumables within a realistic budget for at least one future project.

\section{CONCLUSIONS}

The three principles of K-12 engineering education, namely, design, science knowledge, and mindset development have been implemented in a College Now outreach program designed for high school students. The 
Microsoft Excel enabled exercises in project-based learning strategy can be evaluated with latent variable models. Knowing that smartphone radiation may reduce figural memory capacity in the studied adolescents, a latent variable assessment model could include figural memory scores as data (Ref 44, 45). Future studies could include an investigation of the effectiveness of the integration of project-based learning and applied research into Teacher Professional Development Programs, the development of a SAT II high school exam on engineering, etc.

\section{ACKNOWLEDGEMENTS}

The authors thank Alexei Kisselev for demonstration support and the Muon and Neutron Monitoring Stations for sharing their data in the Public Domain. The authors thank Eric Cheung MD UIC Medical College Psychiatry Department for neuroscience discussion. (S.D. and T.C. wrote the manuscript with inputs from all authors.)

\section{REFERENCES}

1. Dimock, M., "Defining generations: Where Millennials end and post-Millennials begin", Pew Research Center, March (2018) http://www.pewresearch.org/fact-tank/2018/03/01/defining-generations-wheremillennials-end-and-post-millennials-begin/

2. Goodman, M.J. , Sands, A.M., Coley, R.J., “AMERICA’S SKILLS CHALLENGE: Millennials and the Future", Educational Testing Service (2015)

http://www.ets.org/s/research/30079/index.html

3. Barnes \& Noble College, "Getting to Know Gen Z - Exploring Middle and High Schoolers' Expectations for Higher Education", B\&N report (2015) https://next.bncollege.com/wpcontent/uploads/2015/10/Gen-Z-Research-Report-Final.pdf

4. CNW News, "Automation to impact at least $50 \%$ of Canadian jobs in the next decade: RBC research". March 26 (2018). https://www.newswire.ca/news-releases/automation-to-impact-at-least-50-ofcanadian-jobs-in-the-next-decade-rbc-research-677900483.html

5. Mckay, D., "The coming skill revolution: Human Wanted" Royal Bank of Canada (2018) https://www.rbc.com/dms/enterprise/futurelaunch/_assets-custom/pdf/RBC-Future-Skills-ReportFINAL-Singles.pdf

6. Katehi, L., "Engineering in K-12 Education before the Subcommittee on Research and Science Education Committee on Science" U.S. House of Representatives, (2009) https://science.house.gov/sites/republicans.science.house.gov/files/documents/hearings/102209_Katehi. pdf

7. LISREL9.30 student version. http://www.ssicentral.com/lisrel/student.html

8. Dehipawala, S., et al. "An experiential learning strategy in introductory mechanics using transferrable knowledge from daily examples and feedback inquiry in the development of an innovative mindset". ASEE Mid-Atlantic Spring Conference Proceedings (2018) https://peer.asee.org/29453

9. Dehipawala, S., et al. "Developing an engineering mindset: teacher enrichment strategies employing project-based learning in optics and cosmic ray study", ASEE Mid-Atlantic Spring Conference Proceedings (2018). https://peer.asee.org/29459

10. Stanford University. "Magnetism on the Sun- magnetic reconnection". Stanford Solar Center (2017) http://solar-center.stanford.edu/magnetism/magnetismsun.html

11. Li, Y., et al, "Observations of an X-shaped Ribbon Flare in the Sun and Its Three-dimensional Magnetic Reconnection". ApJL, 823, L13 (2016) (arxiv.org/abs/1605.01833)

12. Li., Y. "Observations of an X-shaped-ribbon Flare and Its Three-dimensional Magnetic Reconnection". HMI News Nugget (2016) s http://hmi.stanford.edu/hminuggets/?p=1552

13. Lonecope, D.W. et al, "Effects of Partitioning and extrapolation on the connectivity of potential magnetic fields". ApJL, 693, L1 (2009) (iopscience.iop.org/article/10.1088/0004-637X/693/1/97)

14. Li. Y. , "Movie of X-shaped Ribbon Flare of 2014 November 9" (2016) http://solar.physics.montana.edu/yingli/20141109/HMInugget/index.html 
15. NIH ImageJ, www.imagej.nih.gov/ij

16. Ritter, A.T., et al, "Actin depletion initiates events leading to granule secretion at the immunological synapse", Immunity. Vol 42(5):864-76 (2015) (pubmed/25992860)

17. Killer T-cell video site, https://www.cimr.cam.ac.uk/research/principal-investigators/principalinvestigators-a-h/griffiths

18. Subramaniam, R., Sullivan, R., Schneider, P.S., Holden, T., Tremberger, G, et al, "Intensity fluctuation analysis of cell scattering/imaging with clinical application". Proc. SPIE 6796, 67960N (12 pages), editor: John Armitage (2007)

19. Wadkin L.E. et al. "Correlated random walks of human embryonic stem cells in vitro". Phys Biol. Vol 15(5):056006 (2018) https://www.ncbi.nlm.nih.gov/pubmed/29897045 (arxiv/1803.00063)

20. Wadkin L.E. et al. "Dynamics of single human embryonic stem cells and their pairs: a quantitative analysis". Sci Rep. Vol 7(1):570 (2017). https://www.ncbi.nlm.nih.gov/pubmed/28373677

21. Yao, H. et al. "Leukaemia hijacks a neural mechanism to invade the central nervous system". Nature. Published July 18 2018. https://www.nature.com/articles/s41586-018-0342-5

22. Ashdown G.W. and Owen, D.M., "Spatio-temporal image correlation spectroscopy and superresolution microscopy to quantify molecular dynamics in T cells." Methods. Vol 140-141:112-118 (2018) (pubmed/29410223)

23. Newcastle University Nikon Biostation Live Cell Imaging 2017. youtube.com/watch? $\mathrm{v}=\mathrm{ybBaCYeck} 0 \mathrm{I}$

24. Centeno, C, "How to Determine Cell Viability and Vitality", August 8 2017. regenexx.com/cellviability-cell-vitality/

25. Thermo-Fisher, LIVE/DEAD Cell Vitality Assay Kit, thermofisher.com/order/catalog/product/L34951

26. Zhang, Y., et al. "Influences of acid-treated multiwalled carbon nanotubes on fibroblasts: proliferation, adhesion, migration, and wound healing." Ann Biomed Eng. Vol 39(1), pp 414-26. (2011). https://www.ncbi.nlm.nih.gov/pubmed/20824344

27. Kokkinos, D., Holden, T., Dehipawala, S., et al. "Single mode and sub-cellular fiber probes for cell scattering and density variation measurements" SPIE Proceedings Vol. 8230 Biomedical Applications of Light Scattering VI, Edited by Adam P. Wax and Vadim Backman, 82301G, (2012)

28. Shekoyan, V., et al. "Latent variable modeling with applications to education assessment and NSF-REU projects for engineering students". ASEE Mid-Atlantic Fall Conference Proceedings. (2017) https://peer.asee.org/29384

29. Chu, J. "Physicists design $\$ 100$ handheld muon detector". MIT News Office Nov 20 (2017) http://news.mit.edu/2017/handheld-muon-detector-1121

30. Axani, S.N., et al. "The desktop muon detector: A simple, physics-motivated machine- and electronicsshop project for university students". American Journal of Physics 85, 948 (2017) https://aapt.scitation.org/doi/10.1119/1.5003806

31. Armendariz, R., et al. "Design and construction of a cosmic ray detector array for undergraduate research at the City University of New York". ASEE Mid-Atlantic Fall Conference Proceedings (2017) https://peer.asee.org/29372

32. Armendariz, R., et al. "Cosmic ray detection and magnetic cloud volatility analysis suitable for high school student research" ASEE Mid-Atlantic Fall Conference Proceedings. (2017) https://peer.asee.org/29371

33. Yale University Fractal website. "Power law relations", last accessed July 25 (2018) https://users.math.yale.edu/public_html/People/frame/Fractals/FracAndDim/BoxDim/PowerLaw/Power Law.html

34. V Springel, et al. , "First results from the IllustrisTNG simulations: matter and galaxy clustering", Monthly Notices of the Royal Astronomical Society, Volume 475, Issue 1, 21 March 2018, Pages 676698 (arxiv.org/abs/1707.03397)

35. Liu-Chittenden, Y. et al. "Genetic and pharmacological disruption of the TEAD-YAP complex suppresses the oncogenic activity of YAP." Genes Dev. Vol 26(12):1300-5 (2012) https://www.ncbi.nlm.nih.gov/pubmed/22677547

36. Brown, S., "The Science and Application of Hematoxylin and Eosin Staining" Northwestern University Mouse Histology \& Phenotyping Laboratory Presentations 2012 
http://mhpl.facilities.northwestern.edu/files/2013/10/The-Science-and-Application-of-Hematoxylinand-Eosin-Staining-6-5-2012.pdf

37. Chaelin, K., et al. "Association of Digital Media Use With Subsequent Symptoms of AttentionDeficit/Hyperactivity Disorder Among Adolescents”. JAMA Vol 320(3):255-263 (2018) https://jamanetwork.com/journals/jama/article-abstract/2687861

38. Jiang B., Schmillen, R., Sullivan, W.C.,"How to Waste a Break: Using Portable Electronic Devices Substantially Counteracts Attention Enhancement Effects of Green Spaces. Environment and Behavior". Published July 16, 2018. http://journals.sagepub.com/doi/10.1177/0013916518788603

39. CMS-HCAL collaboration "Brightness and uniformity measurements of plastic scintillator tiles at the CERN H2 test beam" CERN, Nov 302017 https://arxiv.org/abs/1709.08672

40. Pohl, R., "Low energy negative muon beam" Max Planck Institute, 2018 July http://www2.mpq.mpg.de/ rnp/wiki/pmwiki.php/Research/MuonBeam

41. Muon g-2 Experiment Fermi Lab. http://muon-g-2.fnal.gov/

42. Shekoyan, V., et al. "Linking facts with judgment: a critical thinking component in introductory calculus physics for engineering students" ASEE Mid-Atlantic Fall Conference Proceedings. (2017) https://peer.asee.org/29385

43. Wragg, P., et al. "Comparison of Biolog GEN III MicroStation semi-automated bacterial identification system with matrix-assisted laser desorption ionization-time of flight mass spectrometry and $16 \mathrm{~S}$ ribosomal RNA gene sequencing for the identification of bacteria of veterinary interest" Journal of Microbiological Methods Volume 105, pages 16-21 (2014) https://www.sciencedirect.com/science/article/pii/S0167701214001882

44. Swiss Tropical and Public Health Institute. "Mobile Phone Radiation may Affect Memory Performance in Adolescents". July 19 2018. https://www.swisstph.ch/en/news/news-detail/news/mobile-phoneradiation-may-affect-memory-performance-in-adolescents/

45. Foerster M., Thielens A., Joseph W., Eeftens M., Röösli M. A prospective cohort study of adolescents' memory performance and individual brain dose of microwave radiation from wireless communication. Environmental Health Perspectives. Environmental Health Perspective 2018, in press https://www.swisstph.ch/fileadmin/user_upload/EHP2427_DRAFT.pdf 